\title{
Robust all-source positioning of UAVs based on belief propagation
}

\author{
Xi Chen ${ }^{*}$, Wenyun Gao and Jiabo Wang
}

\begin{abstract}
For unmanned air vehicles (UAVs) to survive hostile operational environments, it is always preferable to utilize all wireless positioning sources available to fuse a robust position. While belief propagation is a well-established method for all source data fusion, it is not an easy job to handle all the mathematics therein. In this work, a comprehensive mathematical framework for belief propagation-based all-source positioning of UAVs is developed, taking wireless sources including Global Navigation Satellite Systems (GNSS) space vehicles, peer UAVs, ground control stations, and signal of opportunities. Based on the mathematical framework, a positioning algorithm named Belief propagationbased Opportunistic Positioning of UAVs (BOPU) is proposed, with an unscented particle filter for Bayesian approximation. The robustness of the proposed BOPU is evaluated by a fictitious scenario that a group of formation flying UAVs encounter GNSS countermeasures en route. Four different configurations of measurements availability are simulated. The results show that the performance of BOPU varies only slightly with different measurements availability.
\end{abstract}

Keywords: UAV; Cooperative positioning; Belief propagation; Unscented particle filter

\section{Introduction}

Unmanned air vehicles (UAVs) require an accurate estimate of their positions, velocities, and attitudes in order to control themselves, navigate, and reason about their environment. The way this is achieved varies greatly from systems to systems. While most current UAV navigation systems rely on a combination of the Global Navigation Satellite Systems (GNSS) and an inertial measurement unit (IMU), there is a trend towards the use of all navigation sources available to meet the endless pursuit of navigation robustness in the face of new threats and mission challenges [1-3].

For UAVs, besides GNSS signals, ranging with ground control stations and peer UAVs is readily achievable. Recently, signals of opportunities (SoOPs) from existing RF background infrastructure, such as digital terrestrial wireless TV signal, have also aroused much interests in the research community [4-6]. Under such a context, an innovative positioning algorithm is needed to fuse a right position utilizing all these measurements.

Existing positioning algorithms in the literature are enormous. Early pseudorange-only positioning algorithms for

*Correspondence: chenxiee@tsinghua.edu.cn

Space Center, Tsinghua University, Haidian District, Beijing, 100084, China
GNSS were based on iterative least square and Kalman filtering $[7,8]$. Extended Kalman filtering is a natural extension to Kalman filtering for solving nonlinear problems using one-order linearization [9]. A big step forward for Kalman-based filtering is the invention of unscented Kalman filtering [10-12]. In cooperative positioning [13-20], nodes have not only pseudoranges from navigation satellites but also ranging information with wireless peers. Existing algorithms such as iterative least square and Kalman filters can be extended to cooperative positioning, which leads to cooperative least square and cooperative Kalman filtering algorithm [21]. In recent years, convex optimizations, including semidefinite programming (SDP) [22-25], sum of squares (SOS) relaxation [26,27], and distributed gradient algorithm, also found their place in cooperative positioning. Another category of positioning algorithms are Bayesian approaches with belief propagation as an outstanding representative. Belief propagation-based cooperative positioning was studied for wireless sensor networks, mobile ad hoc networks, vehicle ad hoc networks, etc. [28-31]. By jointly using ranges with peer nodes and pseudoranges from satellites, cooperative positioning dramatically improves the availability and accuracy of positioning, especially in GNSS-challenged environments.

\section{Springer}

(C) 2013 Chen et al.; licensee Springer. This is an Open Access article distributed under the terms of the Creative Commons Attribution License (http://creativecommons.org/licenses/by/2.0), which permits unrestricted use, distribution, and reproduction in any medium, provided the original work is properly cited. 
Despite that positioning has been treated in various settings, there lack a study for positioning of UAVs with all wireless sources available, which is of fatal importance for UAVs to survive hostile operational environments. In fact, the US Defense Advanced Research Projects Agency (DARPA) had already initiated its All Source Positioning and Navigation (ASPN) program as emerging threat scenarios become more sophisticated and widespread. Under all-source positioning context, flexibility and robustness, which allow for run-time join and leave of measurements, are of the first concern. Accuracy, however, is of the second concern. In existing work, algorithms based on belief propagations have proved to be the best candidate to meet the above requirements. While belief propagation is a well-established algorithm, it is not an easy job to handle the mathematics therein when all wireless positioning sources are considered.

For the above motivations, all-source positioning of UAVs based on belief propagations is studied in this work. The positioning sources include (1) pseudoranges and carrier phases from GNSS satellites, (2) ranges and closed-loop Doppler from peer UAVs, (3) ranging information and closed-loop Doppler with ground control stations [32], and (4) time difference of arrival (TDoA) from the signal of opportunities of background wireless infrastructure. The contributions are as follows: (1) A unified mathematical framework for position and velocity estimation is developed, taking all the above measurements and their statistical characteristics. (2) Based on the mathematical framework, a positioning algorithm, named Belief propagation-based Opportunistic Positioning of UAVs (BOPU), is proposed. (3) The factor products, which are mathematically intractable, are solved by an unscented particle filtering. For the accuracy performance of belief propagation with particle filters over Kalman filters and cooperative least square algorithms has already been proved by existing work such as [28,29], we focused on evaluating the robustness of BOPU. Simulations are conducted with a fictitious scenario that a group of formation flying UAVs are under the support of ground control stations but encounter GNSS countermeasures en route. Four different configurations of measurements are simulated and compared. The results show that the performance of BOPU varies only slightly with measurements availability.

The rest of the paper is organized as follows: Section 2 formulates the problem, Section 3 gives the details of the proposed positioning algorithm, Section 4 presents simulation results and discussions, and Section 5 concludes the paper.

\section{Problem formulation}

Consider a group of formation flying UAVs that are carrying out a mission. All UAVs, together with their ground control stations, form a wireless network. The set of UAVs is defined by a wireless node set $\mathbf{M}$ of cardinality $|\mathbf{M}|$. Without loss of generosity, it is assumed that only one GNSS constellation is available with a set of satellites $\mathbf{S}$ of cardinality $|\mathbf{S}|$. There is also a set of SoOP signal sources $\mathbf{G}$ of cardinality $|\mathbf{G}|$. The epoch sequence is denoted by $t^{(0)}, t^{(1)}, \ldots, t^{(k)}$. For a selected wireless node $m \in \mathbf{M}$, denote by $\mathbf{M}_{m}^{(k)}$ the nodes that node $m$ ranges with, by $\mathbf{S}_{m}^{(k)}$ the subset of satellites $m$ is in view, and by $\mathbf{G}_{m}^{(g, k)}$ the nodes that $m$ shares TDoA about SoOP signal source $g \in \mathbf{G}$. The location of node $m$ at epoch $k$ is denoted by $\boldsymbol{\ell}_{m}^{(k)}=\left[\ell_{m x}^{(k)}, \ell_{m y}^{(k)}, \ell_{m z}^{(k)}\right]^{T}$. The velocity of node $m$ at epoch $k$ is denoted by $\boldsymbol{v}_{m}^{(k)}=\left[v_{m x}^{(k)}, v_{m y}^{(k)}, v_{m z}^{(k)}\right]^{T}$. The clock bias of node $m$ with reference to the selected GNSS constellation is denoted as $b_{m}^{(k)}$ in meters, which notionally equals the product of the speed of light multiplying the clock bias of node $m$ in seconds. Define the state of node $m$ as the vector $\mathbf{x}_{m}^{(k)} \triangleq\left[\left(\boldsymbol{\ell}_{m}^{(k)}\right)^{T},\left(\mathbf{v}_{m}^{(k)}\right)^{T}, b_{m}^{(k)}\right]^{T}$. Node $m$ performs the following measurements:

1. Pseudorange $\rho_{s \rightarrow m}^{(k)}$ from satellite $s \in S$, which is in the form

$$
\rho_{s \rightarrow m}^{(k)}=\left\|\boldsymbol{\ell}_{s}^{(k)}-\boldsymbol{\ell}_{m}^{(k)}\right\|+b_{m}^{(k)}+b_{s}^{(k)}+\varepsilon_{\rho s}^{(k)}
$$

where $b_{s}^{(k)}$ represents the sum of correlated errors which generally include tropospheric error, ionospheric error, and ephemeris error, while $\varepsilon_{\rho s}^{(k)}$ represents all uncorrelated errors following a Gaussian distribution.

2. Carrier phase elapsed $\phi_{s \rightarrow m}^{(k)}$ during epoch $k$ and $k-1$ from satellite $s \in S$, which is in the form

$$
\phi_{s \rightarrow m}^{(k)}=|\phi|_{m}+\varepsilon_{\phi s}^{(k)}
$$

where $|\phi|_{m}$ is the true value and $\varepsilon_{\phi s}^{(k)}$ is the carrier phase observation Gaussian error.

3. Ranges $r_{n \leftrightarrow m}^{(k)}$ with neighbors $n \in \mathbf{M}_{m}^{(k)}$, which is in the form

$$
r_{n \leftrightarrow m}^{(k)}=\left\|\boldsymbol{\ell}_{n}^{(k)}-\boldsymbol{\ell}_{m}^{(k)}\right\|+\varepsilon_{r}^{(k)}
$$

where $n$ is the index of the neighbor and $\varepsilon_{r}^{(k)}$ is the ranging error following a Gaussian distribution. A neighbor can be a peer UAV or a ground control station. The position of a UAV is unknown, but that of a ground control station is assumed to be known. Note $r_{n \leftrightarrow m}^{(k)}=r_{m \leftrightarrow n}^{(k)}$.

4. Closed-loop Doppler measurement $f_{n \leftrightarrow m}^{(k)}$ with neighbors $n \in \mathbf{M}_{m}^{(k)}$. By closed-loop Doppler measurement [32], the clock differential of wireless neighbors is eliminated and the resulting $f_{n \leftrightarrow m}^{(k)}$ contains the Doppler that is only related to the 
relative movement of the neighbor nodes involved, specifically,

$$
f_{n \leftrightarrow m}^{(k)}=\frac{\left(\boldsymbol{v}_{n}^{(k)}-\boldsymbol{v}_{m}^{(k)}\right) \bullet \mathbf{1}_{n \rightarrow m}}{\lambda}+\varepsilon_{f}^{(k)}
$$

where $\lambda$ is the wavelength of the carrier used in Doppler measurement, and

$$
\mathbf{1}_{n \rightarrow m} \triangleq \frac{\left(\boldsymbol{\ell}_{n}^{(k)}-\boldsymbol{\ell}_{m}^{(k)}\right)}{\left\|\boldsymbol{\ell}_{n}^{(k)}-\boldsymbol{\ell}_{m}^{(k)}\right\|}
$$

From Equation 4, we have $f_{n \leftrightarrow m}^{(k)}=f_{m \leftrightarrow n}^{(k)}$.

5. TDoA $d_{n \rightarrow m}^{(g, k)}$ in meters with neighbors $n \in \mathbf{G}_{m}^{(g, k)}$ referring to $g$, which is in the form

$$
d_{n \rightarrow m}^{(k)}=\left(\left\|\ell_{g}^{(k)}-\boldsymbol{\ell}_{n}^{(k)}\right\|-\left\|\boldsymbol{\ell}_{g}^{(k)}-\boldsymbol{\ell}_{m}^{(k)}\right\|\right)+\varepsilon_{d}^{(k)}
$$

In (6), $c$ is the speed of light and $\varepsilon_{d}^{(k)}$ is the error in meters, following a Gaussian distribution. Attaining TDoA measurement requires synchronization of nodes $n$ and $m$. Nowadays, one way to achieve this is to use high-quality atomic clock re-synchronized at every departure. In such case, $\varepsilon_{d}^{(k)}$ increases slowly as clock drift accumulates with time.

For brevity, we further define the following notations: $\tilde{\boldsymbol{X}}_{M}^{(k)} \triangleq\left\{\boldsymbol{x}_{m}^{(k)} \mid \forall m \in \boldsymbol{M}\right\} ; \boldsymbol{P}_{m}^{(k)} \triangleq\left\{\rho_{s \rightarrow m}^{(k)} \mid \forall s \in \boldsymbol{S}_{m}^{(k)}\right\}$, $\tilde{\boldsymbol{P}}_{M}^{(k)} \triangleq\left\{\boldsymbol{P}_{m}^{(k)} \mid \forall m \in \boldsymbol{M}\right\} ; \boldsymbol{R}_{m}^{(k)} \triangleq\left\{r_{n \leftrightarrow m}^{(k)} \mid \forall n \in \boldsymbol{M}_{m}^{(k)}\right\}$, $\tilde{\boldsymbol{R}}_{M}^{(k)} \triangleq\left\{\boldsymbol{R}_{m}^{(k)} \mid \forall m \in \boldsymbol{M}\right\} ; \boldsymbol{F}_{m}^{(k)} \triangleq\left\{f_{n \leftrightarrow m}^{(k)} \mid \forall n \in \boldsymbol{M}_{m}^{(k)}\right\}$, $\tilde{\boldsymbol{F}}_{m}^{(k)} \triangleq\left\{\boldsymbol{F}_{M}^{(k)} \mid \forall m \in \boldsymbol{M}\right\} ; \boldsymbol{D}_{m}^{(g, k)} \triangleq\left\{d_{n \rightarrow m}^{(g, k)} \mid \forall n \in \boldsymbol{G}_{m}^{(g, k)}\right\}$, $\tilde{\boldsymbol{D}}_{M}^{(g, k)} \triangleq\left\{\boldsymbol{D}_{m}^{(g, k)} \mid \forall m \in \boldsymbol{M}\right\}, \tilde{\boldsymbol{D}}_{M}^{(k)} \triangleq\left\{\tilde{\boldsymbol{D}}_{M}^{(g, k)} \mid \forall g \in \mathbf{G}\right\} ;$ $\boldsymbol{\Phi}_{m}^{(k)} \triangleq\left\{\varphi_{s \rightarrow m}^{(k)} \mid \forall s \in \boldsymbol{S}_{m}^{(k)}\right\}, \tilde{\boldsymbol{\Phi}}_{M}^{(k)} \triangleq\left\{\boldsymbol{\Phi}_{m}^{(k)} \mid \forall m \in \boldsymbol{M}\right\} ;$ $\tilde{\boldsymbol{O}}_{M}^{(k)}=\left\{\tilde{\boldsymbol{P}}_{M}^{(1: k)}, \tilde{\boldsymbol{R}}_{M}^{(1: k)}, \tilde{\boldsymbol{D}}_{M}^{(k)}, \tilde{\boldsymbol{\Phi}}_{M}^{(1: k)}, \tilde{\boldsymbol{F}}_{M}^{(1: k)\}}\right\}$.

The goal of the positioning is to find the a posteriori distribution of $\boldsymbol{x}_{m}^{(k)}$ at each epoch $k$, given all the available observations $\tilde{\boldsymbol{O}}_{M}^{(k)}$ :

$$
p\left(\boldsymbol{x}_{m}^{(k)} \mid \tilde{\boldsymbol{O}}_{M}^{(1: k)}\right), \forall m \in \boldsymbol{M}
$$

where $(1: k)$ denotes the epochs from 1 to $k$. At epoch $k$, the final estimation $\boldsymbol{\mu}_{m}^{(k)}$ is the statistical expectation of $\boldsymbol{x}_{m}^{(k)}$ as

$$
\boldsymbol{\mu}_{m}^{(k)}=\int \boldsymbol{x}_{m}^{(k)} p\left(\boldsymbol{x}_{m}^{(k)} \mid \tilde{\boldsymbol{O}}_{M}^{(k)}\right)
$$

\section{The proposed BOPU}

\subsection{The Bayesian inference}

It is reasonable to assume that ranges with peer UAVs and the control stations are independent, and it is also often the case that the nodes in $\boldsymbol{M}$ move independently. The pseudoranges are independent when ignoring $b_{s}^{(k)}$.
The error induced by ignoring $b_{s}^{(k)}$ will be discussed later. While the movement of a node can be measured readily by IMUs in many cases, it is not the case in all-source positioning because IMUs and wireless measurement are loosely coupled for flexibility. In this work, the movement of node $m \in M$ is modeled as a second-order Markov process. With these assumptions, (7) can be rewritten as

$$
p\left(\boldsymbol{x}_{m}^{(k)} \mid \tilde{\boldsymbol{O}}_{M}^{(1: k)}\right)=\int p\left(\tilde{\boldsymbol{X}}_{M}^{(k-2: k)} \mid \tilde{\boldsymbol{O}}_{M}^{(1: k)}\right) \partial \tilde{\boldsymbol{X}}_{M \backslash m}^{(k-2: k)}
$$

where $M \backslash m$ denotes all variables in $\tilde{\boldsymbol{X}}_{M}^{(k-1: k)}$ except $\boldsymbol{x}_{m}^{(k)}$. To determine the marginal (7) recursively at each epoch $k$, the integrand of (9) can be further decomposed as

$$
\begin{aligned}
& p\left(\tilde{\boldsymbol{X}}_{M}^{(k-2: k)} \mid \tilde{\boldsymbol{O}}_{M}^{(1: k)}\right) \propto p\left(\tilde{\boldsymbol{P}}_{M}^{(k)} \mid \tilde{\boldsymbol{X}}_{M}^{(k)}, \tilde{\boldsymbol{\Phi}}_{M}^{(1: k)}\right) \times \\
& p\left(\tilde{\boldsymbol{R}}_{M}^{(k)} \mid \tilde{\boldsymbol{X}}_{M}^{(k)}, \tilde{\boldsymbol{F}}_{M}^{(1: k)}\right) p\left(\tilde{\boldsymbol{G}}_{M}^{(k)} \mid \tilde{\boldsymbol{X}}_{M}^{(k)}\right) \times \\
& p\left(\tilde{\boldsymbol{\Phi}}_{M}^{(k)} \mid \tilde{\boldsymbol{X}}_{M}^{(k)}\right) p\left(\tilde{\boldsymbol{F}}_{M}^{(k)} \mid \tilde{\boldsymbol{X}}_{M}^{(k)}\right) \times \\
& \left.\prod_{m \in \boldsymbol{M}} p\left(\boldsymbol{x}_{m}^{(k)} \mid \boldsymbol{x}_{m}^{(k-2: k-1)}\right) p\left(\boldsymbol{x}_{m}^{(k-2: k-1)} \mid \tilde{\boldsymbol{O}}_{M}^{(1: k-1)}\right)\right)
\end{aligned}
$$

Ignoring the backward smoothing of $\tilde{\boldsymbol{O}}_{M}^{(k-1)}$ on $\boldsymbol{x}_{m}^{(k-2)}$, it holds that

$$
\begin{aligned}
p\left(\boldsymbol{x}_{m}^{(k-2: k-1)} \mid \tilde{\boldsymbol{O}}_{M}^{(1: k-1)}\right)= & p\left(\boldsymbol{x}_{m}^{(k-1)} \mid \tilde{\boldsymbol{O}}_{M}^{(k-1)}\right) \\
& \times p\left(\boldsymbol{x}_{m}^{(k-2)} \mid \tilde{\boldsymbol{O}}_{M}^{(k-2)}\right)
\end{aligned}
$$

Given the state $\tilde{\boldsymbol{X}}_{M}^{(k)}$, all the measurements are statistically independent, so

$$
p\left(\tilde{\boldsymbol{P}}_{M}^{(k)} \mid \tilde{\boldsymbol{X}}_{M}^{(k)}, \tilde{\boldsymbol{\Phi}}_{M}^{(1: k)}\right)=\prod_{m \in M_{s \in \boldsymbol{S}_{m}^{(k)}}} \prod_{\rho_{s \rightarrow m}} p\left(\boldsymbol{x}_{m}^{(k)}, \phi_{s \rightarrow m}^{(1: k)}\right)
$$

$$
\begin{aligned}
& p\left(\tilde{\boldsymbol{R}}_{M}^{(k)} \mid \tilde{\boldsymbol{X}}_{M}^{(k)}, \tilde{\boldsymbol{F}}_{M}^{(1: k)}\right) \\
& =\prod_{m \in \boldsymbol{M}_{n \in \boldsymbol{M}_{m}^{(k)}, n>m}} \prod_{n} p\left(r_{n \leftrightarrow m}^{(k)} \mid \boldsymbol{x}_{m}^{(k)}, \boldsymbol{x}_{n}^{(k)}, f_{n \leftrightarrow m}^{(1: k)}\right) \\
& p\left(\tilde{\boldsymbol{G}}_{M}^{(k)} \mid \tilde{\boldsymbol{X}}_{M}^{(k)}\right)=\prod_{g \in \boldsymbol{G}} \prod_{m \in \boldsymbol{M}_{n \in \boldsymbol{G}_{m}^{(g, k)}, n>m}} p\left(d_{n \rightarrow m}^{(g, k)} \mid \boldsymbol{x}_{m}^{(k)}, \boldsymbol{x}_{n}^{(k)}\right)
\end{aligned}
$$

$$
\begin{aligned}
& p\left(\tilde{\boldsymbol{\Phi}}_{m}^{(k)} \mid \tilde{\boldsymbol{X}}_{M}^{(k)}\right)=\prod_{m \in \boldsymbol{M} \in \boldsymbol{S}_{m}^{(k)}} \prod_{\phi_{s \rightarrow m}} p\left(\phi_{m}^{(k)}\right) \\
& p\left(\tilde{\boldsymbol{F}}_{m}^{(k)} \mid \tilde{\boldsymbol{X}}_{M}^{(k)}\right)=\prod_{m \in \boldsymbol{M}_{n \in \boldsymbol{M}_{m}^{(k)}, n>m}} p\left(f_{n \leftrightarrow m}^{(k)} \mid \boldsymbol{x}_{m}^{(k)}\right)
\end{aligned}
$$


Now for each node $m \in M$, we define the following:

1. $\Upsilon_{s, m}\left(\boldsymbol{x}_{m}^{(k)}\right) \triangleq p\left(\rho_{s \rightarrow m}^{(k)} \mid \boldsymbol{x}_{m}^{(k)}, \phi_{s \rightarrow m}^{(1: k)}\right)$, denoting the pseudorange measurement model of node $m$ at epoch $k$.

2. $\Theta_{s, m}\left(\boldsymbol{x}_{m}^{(k)}\right) \triangleq p\left(\phi_{s \rightarrow m}^{(k)} \mid \boldsymbol{x}_{m}^{(k)}\right)$, representing carrier phase measurement model of node $m$ at epoch $k$.

3. $\Gamma_{n, m}\left(\boldsymbol{x}_{n}^{(k)}, \boldsymbol{x}_{m}^{(k)}\right) \triangleq p\left(r_{n \leftrightarrow m}^{(k)} \mid \boldsymbol{x}_{m}^{(k)}, \boldsymbol{x}_{n}^{(k)}, f_{n \leftrightarrow m}^{(1: k)}\right)$, denoting the range measurement model of node $m$ with node $n$ at epoch $k$.

4. $\Omega_{n, m}^{g}\left(\boldsymbol{x}_{n}^{(k)}, \boldsymbol{x}_{m}^{(k)}\right) \triangleq p\left(d_{n \rightarrow m}^{(g, k)} \mid \boldsymbol{x}_{m}^{(k)}, \boldsymbol{x}_{n}^{(k)}\right)$, denoting the TDoA measurement model of node $m$ to $n$ with reference to SoOP source $g$ at epoch $k$.

5. $\Psi_{n, m}\left(\boldsymbol{x}_{n}^{(k)}, \boldsymbol{x}_{m}^{(k)}\right) \triangleq p\left(f_{n \leftrightarrow m}^{(k)} \mid \boldsymbol{x}_{m}^{(k)}, \boldsymbol{x}_{n}^{(k)}, f_{n \leftrightarrow m}^{(1: k)}\right)$, denoting the peer-to-peer Doppler measurements of nodes $m$ and $n$ at epoch $k$.

6. $\Delta_{m}\left(\boldsymbol{x}_{m}^{(k)}, \boldsymbol{x}_{m}^{(k-2: k-1)}\right) \triangleq p\left(\boldsymbol{x}_{m}^{(k)} \mid \boldsymbol{x}_{m}^{(k-2: k-1)}\right)$, denoting dead reckoning of node $m$ from epoch $k-2: k-1$ to $k$.

With the above definitions, we have

$$
\begin{aligned}
& p\left(\tilde{\boldsymbol{X}}_{M}^{(k-1: k)} \mid \tilde{\boldsymbol{O}}_{M}^{(1: k)}\right) \propto \prod_{m \in \boldsymbol{M}}\left(\prod_{s \in \boldsymbol{S}_{m}^{(k)}} \Upsilon_{m}\left(\boldsymbol{x}_{m}^{(k)}\right) \times\right. \\
& \prod_{n \in \boldsymbol{M}_{m}^{(k)}, n>m} \Gamma_{n, m}\left(\boldsymbol{x}_{n}^{(k)}, \boldsymbol{x}_{m}^{(k)}\right) \times \\
& \prod_{g \in \boldsymbol{G}} \prod_{n \in \boldsymbol{G}_{m}^{(g, k)}, n>m} \Omega_{n, m}^{g}\left(\boldsymbol{x}_{n}^{(k)}, \boldsymbol{x}_{m}^{(k)}\right) \times \\
& \prod_{s \in \boldsymbol{S}_{m}^{(k)}} \Theta_{s, m}\left(\boldsymbol{x}_{m}^{(k)}\right) \prod_{n \in \boldsymbol{M}_{m}^{(k)}, n>m} \Psi_{n, m}\left(\boldsymbol{x}_{n}^{(k)}, \boldsymbol{x}_{m}^{(k)}\right) \times \\
& \prod_{n \in \boldsymbol{M}_{m}^{(k)}}\left(\Delta_{m}\left(\boldsymbol{x}_{m}^{(k)}, \boldsymbol{x}_{m}^{(k-2: k-1)}\right) p\left(\boldsymbol{x}_{m}^{(k-2: k-1)} \mid \tilde{\boldsymbol{O}}_{M}^{(k-1)}\right)\right)
\end{aligned}
$$

With Equation 17, we have the factor subgraph of node $m$ as given in Figure 1. The factor subgraphs of all nodes $m \in M$, when interconnected, make up the complete factor graph. Figure 2 illustrates the complete factor graph of nodes of the simulation scenario given by Figure 3 .

\subsection{The sum product update rule}

A belief propagation algorithm defines the sum product messages and their update rules over the factor graph. In our case, there are six classes of messages:

1. Dead-reckon message $h_{\Delta_{m} \rightarrow \boldsymbol{x}_{m}}$, associated to the state of node $m$ from epoch $k-2: k-1$ to $k$

2. Satellite pseudorange factor messages $h_{\Upsilon_{s, m} \rightarrow \boldsymbol{x}_{m}}$, associated to the pseudorange measurements, useful only in estimating $\boldsymbol{l}_{m}^{(k)}$ and $b_{m}^{(k)}$
3. Satellite carrier phase factor messages $h_{\Theta_{s, m} \rightarrow x_{m}}$, associated to the pseudorange measurements, useful only in estimating $\boldsymbol{l}_{m}^{(k)}$ and $\boldsymbol{v}_{m}^{(k)}$

4. Messages from range neighbors $h_{\Gamma_{n, m} \rightarrow x_{m}}$, representing the positioning information from neighbors with range and closed-loop Doppler measurements

5. Messages from SoOP neighbors $h_{\Omega_{n, m}^{g} \rightarrow x_{m}}$, representing the positioning information from neighbors with TDoA measurement with reference to the SoOP source $g$

6. Messages to peers $h_{x_{m} \rightarrow x_{n}}$, where $n \in M_{m}^{(k)}$ which node $m$ sends to all neighbors including range neighbors and SoOP neighbors

The proposed positioning algorithm, named BOPU, includes two steps. The first step is to obtain $p\left(\boldsymbol{x}_{m}^{(0)}\right)$ at epoch 0 , which is done by a cooperative least square positioning. The second step is to obtain $p\left(\boldsymbol{x}_{m}^{(k)} \mid \tilde{\boldsymbol{O}}_{M}^{(1: k)}\right)$ at epoch $k \geq 1$. Using all the above message definitions, the sum product update rule of the proposed positioning algorithm can be given as in Algorithm 1.

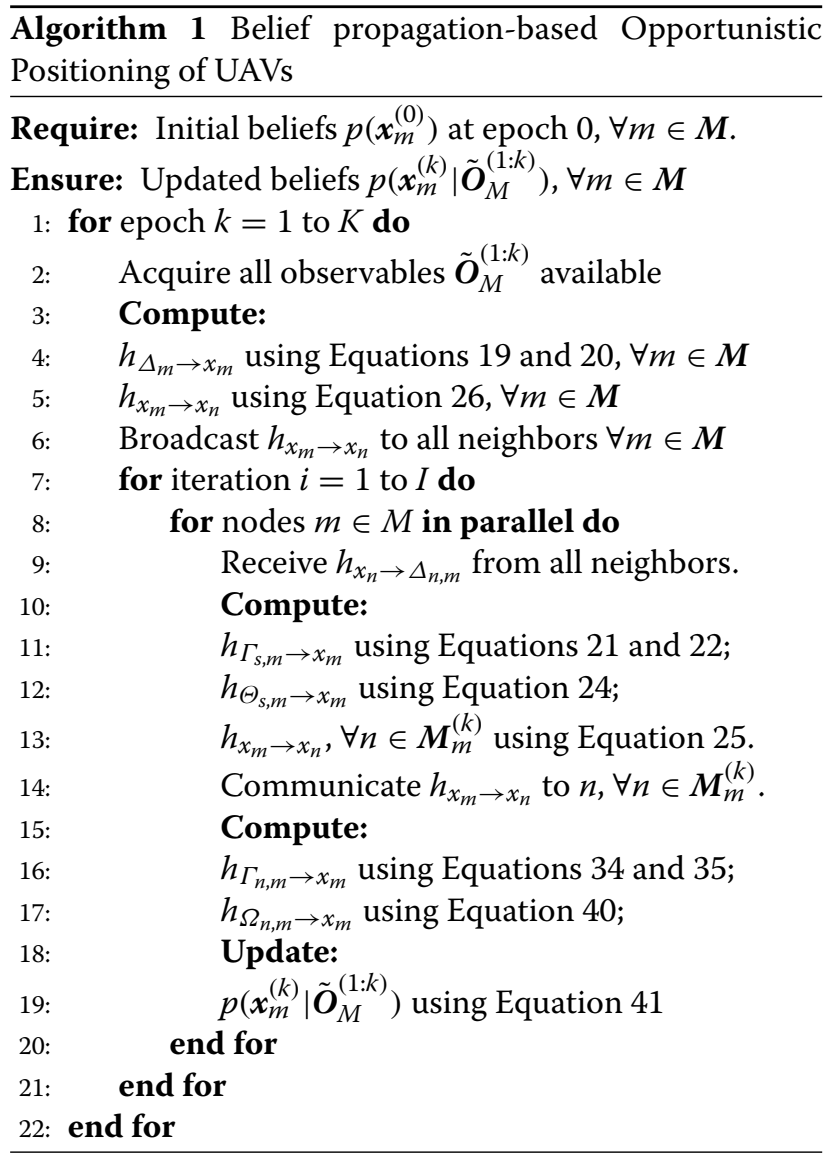




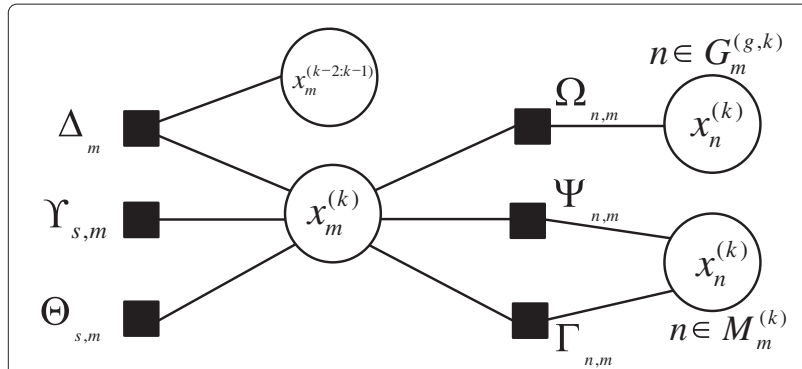

Figure 1 The factor subgraph of node $\boldsymbol{m}$. The factor subgraphs of all nodes $\boldsymbol{m} \in \boldsymbol{M}$ make up the complete factor graph.

\subsection{The messages in parametric form}

A compact parametric form of the messages involved in the proposed algorithm is needed to make it permissible to transmit over a wireless network with limited bandwidth, which are given below:

1. Dead-reckon message $h_{\Delta_{m} \rightarrow x_{m}}$ is associated to the state of node $m$ from epoch $k-2: k-1$ to $k$. From Figure 1, we have

$$
\begin{aligned}
h_{\Delta_{m} \rightarrow x_{m}} \propto & \int \Delta_{m}\left(\boldsymbol{x}_{m}^{(k)}, \boldsymbol{x}_{m}^{(k-2: k-1)}\right) \\
& p\left(\boldsymbol{x}_{m}^{(k-2: k-1)} \mid \tilde{\mathbf{O}}_{M}^{(1: k-1)}\right) \partial \boldsymbol{x}_{m}^{(k-2: k-1)}
\end{aligned}
$$

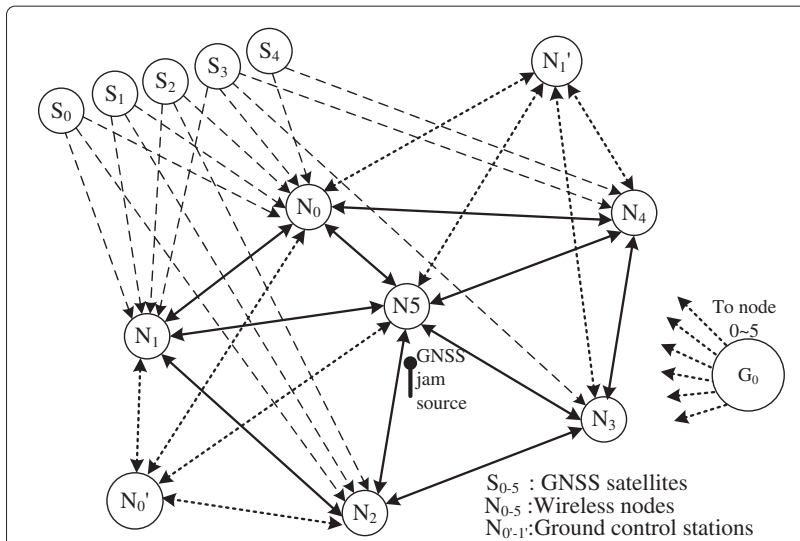

Figure 3 The simulation scenario. A group of six UAVs are flying from a start point under the control of ground control station $N_{0}^{\prime}$ to a destination with ground control station $N_{1}^{\prime}$, but experience a GPS countermeasure in the midway.

From its definition, the dead-reckon message is a Gaussian probability density function with mean $\boldsymbol{\mu}_{x_{m}^{(k)}}$ and covariance $\boldsymbol{\Sigma}_{x_{m}^{(k)}}$. The mean $\boldsymbol{\mu}_{x_{m}^{(k)}}$ and covariance $\boldsymbol{\Sigma}_{x_{m}^{(k)}}$ can be derived from $\boldsymbol{\mu}_{x_{m}^{(k-1)}}$ and $\Sigma_{x_{m}^{(k-1)}}$, respectively. Among the many ways for dead reckoning, we set

$$
\boldsymbol{\mu}_{x_{m}^{(k)}}=2 \boldsymbol{\mu}_{x_{m}^{(k-1)}}-\boldsymbol{\mu}_{x_{m}^{(k-2)}}
$$

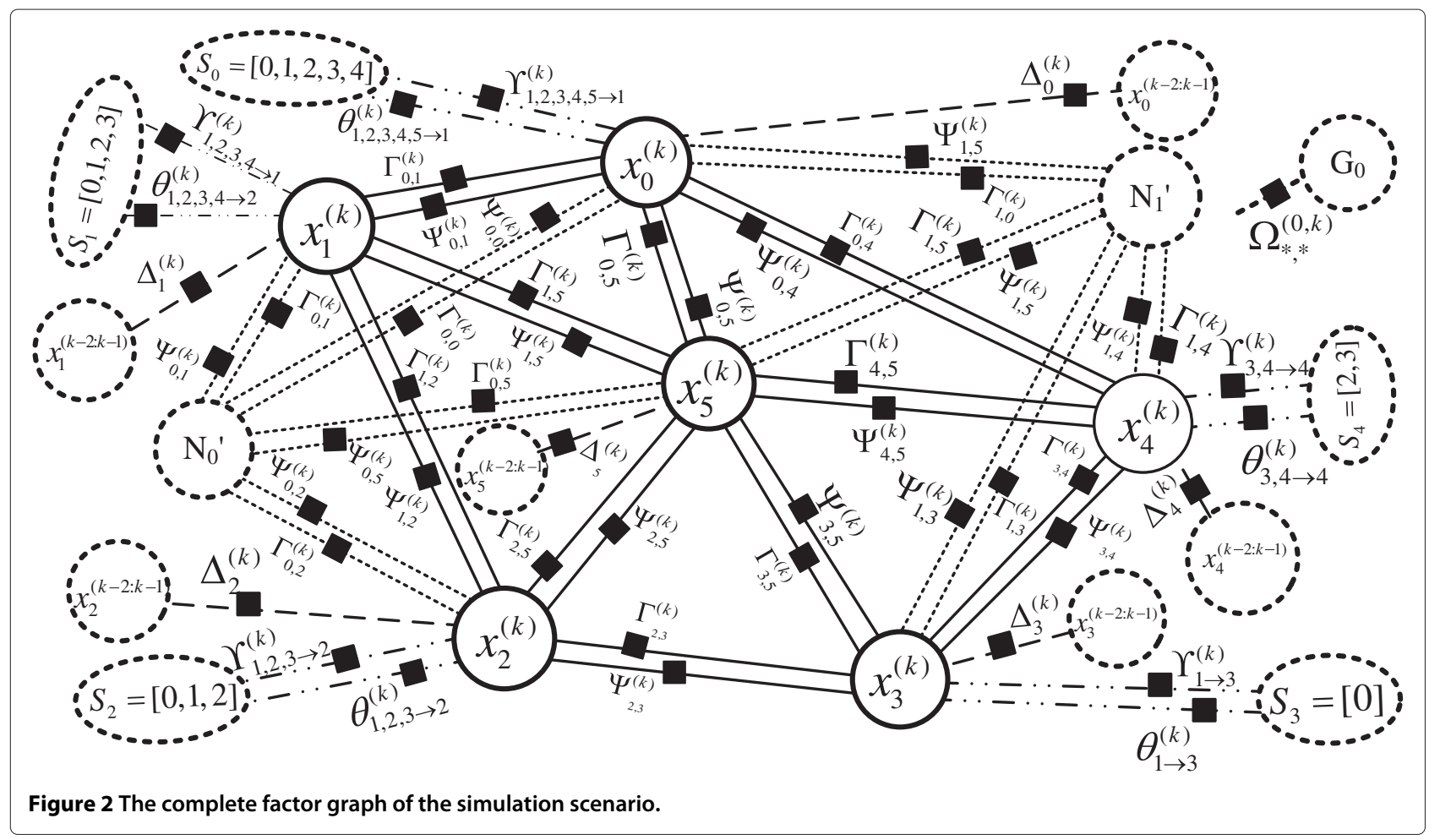


Following (19), it is trivial to derive

$$
\Sigma_{x_{m}^{(k)}}=4 \Sigma_{x_{m}^{(k-1)}}+\Sigma_{x_{m}^{(k-2)}}
$$

2. Satellite factor messages $h_{\Upsilon_{s, m} \rightarrow x_{m}}$ are associated to the pseudoranges of node $m$. The pseudorange of node $m$ from satellite $s$ is assumed to be bias free except the receiver clock bias; thus, we have

$$
h_{\Upsilon_{s, m} \rightarrow x_{m}} \propto \exp \left(-\frac{1}{2 \sigma_{s \rightarrow m}^{2}}\left(\left\|\boldsymbol{l}_{m}^{(k)}-\boldsymbol{l}_{s}^{(k)}\right\|+b_{m}^{(k)}-\hat{\rho}_{s \rightarrow m}^{(k)}\right)^{2}\right)
$$

where $\sigma_{s \rightarrow m}^{2}$ is the pseudorange error power of satellite $s$ at node $m$ and $\hat{\rho}_{s}^{(k)}$ is the carrier phase smoothed pseudorange, which can be expressed in a recursive form as [33]

$$
\hat{\rho}_{s \rightarrow m}^{(k)}=\frac{\rho_{s \rightarrow m}^{(k)}}{k}+\frac{k-1}{k}\left(\hat{\rho}_{s \rightarrow m}^{(k-1)}+\phi_{s \rightarrow m}^{(k)}\right)
$$

We note here that $h_{\Upsilon_{s, m} \rightarrow x_{m}}$ is only contributable to $l_{m}$ and $b_{m}$.

3. Satellite carrier phase factor messages $h_{\Theta_{s, m} \rightarrow x_{m}}$ are associated to satellite carrier phase measurements. For

$$
\left(\lambda \phi_{s \rightarrow m}^{(k)}\right) / T_{e}=\left(\boldsymbol{v}_{s}^{(k)}-\boldsymbol{v}_{m}^{(k)}\right) \bullet \mathbf{1}_{m \rightarrow s}+\delta f_{m}^{(k)}-\delta f_{s}^{(k)}
$$

where $\mathbf{1}_{m \rightarrow s}$ is the unit vector directed from node $m$ to satellite $s, \delta f_{s}^{(k)}$ is the clock drift rate of satellite $s$. So we have

$$
\begin{aligned}
& h_{\Theta_{s, m} \rightarrow x_{m}} \propto \exp \left(\frac { 1 } { 2 \sigma _ { \phi } ^ { 2 } } \left(\phi_{s \rightarrow m}^{(k)}\right.\right. \\
& \left.\left.-\left(\left(v_{s}^{(k)}-v_{m}^{(k)}\right) \bullet \mathbf{1}_{m \rightarrow s}+\delta f_{m}^{(k)}-\delta f_{s}^{(k)}\right) t / \lambda\right)\right)
\end{aligned}
$$

We note here that $h_{\Theta_{s, m} \rightarrow x_{m}}$ is only contributable to $\boldsymbol{v}_{m}$.

4. Messages to peers $h_{x_{m} \rightarrow x_{n}}$ are messages that node $m$ sends to all neighbors, in the following form:

$$
\begin{aligned}
& h_{x_{m} \rightarrow x_{n}} \propto h_{\Delta_{m} \rightarrow x_{m}} \prod_{s \in S_{m}^{(k)}} h_{\Upsilon_{s, m} \rightarrow x_{m}} \times \\
& \prod_{g \in G, n \in G_{m}^{(g, k)}} h_{\Omega_{n, m}^{g} \rightarrow x_{m}} \prod_{s \in S_{m}^{(k)}} h_{\Theta_{s, m} \rightarrow x_{m}} \times \\
& \prod_{n^{\prime} \in M_{m}^{(k)} \backslash n} h_{\Gamma_{n^{\prime}, m} \rightarrow x_{m}}
\end{aligned}
$$

At the initialization stage of each epoch, $h_{\Omega_{h, m}^{g} \rightarrow x_{m}}$ and $h_{\Delta_{n^{\prime}, m} \rightarrow x_{m}}$ are not available; then we have

$$
h_{x_{m} \rightarrow \Delta_{m, n}} \propto h_{\Delta_{m} \rightarrow x_{m}} \prod_{s \in S_{m}^{(k)}} h_{\Upsilon_{s, m} \rightarrow x_{m}} \prod_{s \in S_{m}^{(k)}} h_{\Theta_{s, m} \rightarrow x_{m}}
$$

It is hard to find the exact expression of Equations 25 and 26 , so we approximate the result of message multiplication as a Gaussian distribution:

$$
\begin{aligned}
h_{x_{m} \rightarrow \Delta_{m, n}} \approx & \frac{1}{z} \exp \left[-\frac{1}{2}\left(\boldsymbol{x}_{m}^{(k)}-\boldsymbol{\mu}_{x_{m \rightarrow n}^{(k)}}\right)\right. \\
& \left.\times \boldsymbol{\Sigma}_{x_{m \rightarrow n}^{(k)}}^{-1}\left(\boldsymbol{x}_{m}^{(k)}-\boldsymbol{\mu}_{x_{m \rightarrow n}^{(k)}}\right)^{T}\right]
\end{aligned}
$$

where $z$ is the normalization factor. In practice, the values of $\boldsymbol{\mu}_{x_{m \rightarrow n}^{(k)}}$ and $\boldsymbol{\Sigma}_{x_{m \rightarrow n}^{(k)}}$ are approximated by an unscented particle filtering as Algorithm 2, which will be disposed of later.

5. Messages from range neighbors $h_{\Gamma_{n, m} \rightarrow x_{m}}$ represent the contribution of ranging information from wireless neighbors. For the position of ground control stations are known, $h_{\Gamma_{n, m} \rightarrow x_{m}}$ associated to the ranging information of node $m$ to a control station $n$ can be expressed as

$h_{\Gamma_{n, m} \rightarrow l_{m}} \propto \exp \left(-\frac{1}{2 \sigma_{n \rightarrow m}^{2}}\left(\left\|\boldsymbol{l}_{m}^{(k)}-\boldsymbol{l}_{n}^{(k)}\right\|-\hat{r}_{n \leftrightarrow m}^{(k)}\right)^{2}\right)$

where $\sigma_{n \rightarrow m}^{2}$ is the ranging error power and $\hat{r}_{n}^{(k)}$ is the Doppler smoothed range, which can be expressed in a recursive form as

$\hat{r}_{n \leftrightarrow m}^{(k)}=\frac{r_{n \leftrightarrow m}^{(k)}}{k}+\frac{k-1}{k}\left(\hat{r}_{n \leftrightarrow m}^{(k-1)}+\lambda\left(f_{n \leftrightarrow m}^{(k)}-f_{n \leftrightarrow m}^{(k-1)}\right)\right)$

Ground control stations' Doppler messages $h_{\Psi_{n, m} \rightarrow x_{m}}$ are associated to the Doppler information of node $m$ to ground control stations. Similar to $h_{\Theta_{s, m} \rightarrow x_{m}}$, we have

$$
h_{\Psi_{n, m} \rightarrow \boldsymbol{x}_{m}} \propto \exp \left(\frac{1}{2 \sigma_{f}^{2}}\left(f_{m \leftrightarrow g}^{(k)}+\boldsymbol{v}_{m}^{(k)} \bullet \mathbf{1}_{m \rightarrow n} / \lambda\right)^{2}\right)
$$

Messages from other UAVs whose positions are not known can be expressed as

$$
h_{\Gamma_{n, m} \rightarrow x_{m}} \propto \int \Gamma_{n, m}\left(\boldsymbol{x}_{n}^{(k)}, \boldsymbol{x}_{m}^{(k)}\right) h_{x_{n} \rightarrow x_{m}} \partial \boldsymbol{x}_{n}^{(k)}
$$

To find out the parametric form of this distribution, we follow a divide-and-conquer approach. First, we can see that the mean of position $\boldsymbol{l}_{m}^{(k)}$ in this distribution is a ball with radius $r_{n \leftrightarrow m}^{(k)}$ and center $\boldsymbol{\mu}_{l_{m \rightarrow n}^{(k)}}$, and its covariance is $\boldsymbol{\Sigma}_{x_{n \rightarrow m}^{(k)}}+\sigma_{n \rightarrow m}^{2} \boldsymbol{I}$. Any valid point on the surface of the ball is restricted by

$$
\boldsymbol{\ell}_{r}^{(k)}=\boldsymbol{\mu}_{l_{m \rightarrow n}^{(k)}}+r_{m \rightarrow n}^{(k)} \bullet \mathbf{1}_{n \rightarrow m}^{\prime}
$$


thus, we have

$$
\begin{aligned}
h_{\Gamma_{n, m} \rightarrow l_{m}} \propto \exp [ & -\frac{1}{2}\left(\boldsymbol{l}_{m}^{(k)}-\boldsymbol{\ell}_{r}^{(k)}\right)\left(\boldsymbol{\Sigma}_{x_{m \rightarrow n}^{(k)}}+\sigma_{n \rightarrow m}^{2} \boldsymbol{I}\right)^{-1} \\
& \left.\times\left(\boldsymbol{l}_{m}^{(k)}-\boldsymbol{\ell}_{r}^{(k)}\right)^{T}\right]
\end{aligned}
$$

where

$$
\mathbf{1}_{m \rightarrow n}^{\prime}=\frac{\boldsymbol{l}_{m}^{(k)}-\boldsymbol{\mu}_{\boldsymbol{l}_{m \rightarrow n}^{(k)}}}{\left\|\boldsymbol{l}_{m}^{(k)}-\boldsymbol{\mu}_{l_{m \rightarrow n}^{(k)} \|}\right\|}
$$

Similarly,

$$
\begin{aligned}
h_{\Gamma_{n, m} \rightarrow v_{m}} \propto \exp [ & -\frac{1}{2}\left(\boldsymbol{v}_{m}^{(k)}-\boldsymbol{v}_{r}^{(k)}\right)\left(\boldsymbol{\Sigma}_{v_{m \rightarrow n}^{(k)}}+\sigma_{v}^{2} \boldsymbol{I}\right)^{-1} \\
& \left.\times\left(\boldsymbol{v}_{m}^{(k)}-\boldsymbol{v}_{r}^{(k)}\right)^{T}\right]
\end{aligned}
$$

where

$$
\boldsymbol{v}_{r}=\boldsymbol{\mu}_{v_{m \rightarrow n}^{(k)}}+\lambda f_{m \rightarrow n}^{(k)} \mathbf{1}_{m \rightarrow n}^{\prime}
$$

6. Messages from SoOP neighbors $h_{\Omega_{n, m}^{g} \rightarrow x_{m}}$ represent the contribution of TDoA measurements referencing SoOP source $g$, which can be expressed as

$$
h_{\Omega_{n, m}^{g} \rightarrow x_{m}} \propto \int \Omega_{n, m}^{g}\left(\boldsymbol{x}_{n}^{(k)}, \boldsymbol{x}_{m}^{(k)}\right) h_{x_{n} \rightarrow x_{m}} \partial \boldsymbol{x}_{n}^{(k)}
$$

The mean position $\boldsymbol{l}_{m}^{(k)}$ in this distribution is a ball with center $\boldsymbol{l}_{g}$ and radius $\left\|\boldsymbol{l}_{g}-\boldsymbol{\mu}_{n \rightarrow m}\right\|-d_{n \rightarrow m}^{(k)}$, and its covariance is $\boldsymbol{\Sigma}_{x_{n \rightarrow m}^{(k)}}+\sigma_{d}^{2} \boldsymbol{I}$. We now define a vector

$$
\boldsymbol{\ell}_{g}^{\prime}=\boldsymbol{l}_{m}^{(k)}-\boldsymbol{l}_{g}-\left(d_{m \rightarrow n}^{(k)}+\left\|\boldsymbol{\mu}_{\boldsymbol{l}_{m \rightarrow n}^{(k)}}-\boldsymbol{l}_{g}\right\|\right) \bullet \mathbf{1}_{m \rightarrow g}^{\prime}
$$

where

$$
\mathbf{1}_{m \rightarrow g}^{\prime}=\frac{\boldsymbol{l}_{m}^{(k)}-\boldsymbol{l}_{g}}{\left\|\boldsymbol{l}_{m}^{(k)}-\boldsymbol{l}_{g}\right\|}
$$

thus, we have

$$
h_{\Omega_{n, m} \rightarrow l_{m}} \propto \exp \left(-\frac{1}{2} \boldsymbol{\ell}_{g}^{\prime}\left(\boldsymbol{\Sigma}_{x_{m \rightarrow n}^{(k)}}+\sigma_{d}^{2} \boldsymbol{I}\right)^{-1}\left(\boldsymbol{\ell}_{g}^{\prime}\right)^{T}\right)
$$

Finally, we have $p\left(x_{m}^{(k)} \mid \tilde{\boldsymbol{O}}_{M}^{(1: k)}\right)$ as

$$
\begin{aligned}
& p\left(\boldsymbol{x}_{m}^{(k)} \mid \tilde{\boldsymbol{O}}_{\boldsymbol{M}}^{(1: k)}\right) \propto h_{\Delta_{m} \rightarrow x_{m}} \prod_{s \in \boldsymbol{S}_{m}^{(k)}} h_{\Gamma_{s, m} \rightarrow x_{m}} \\
& \prod_{g \in \boldsymbol{G}_{m}^{(k)}} \prod_{s \in \boldsymbol{S}_{m}^{(k)}} h_{\Theta_{s, m} \rightarrow x_{m}} \prod_{g \in \boldsymbol{G}, n \in \boldsymbol{G}_{m}^{(g, k)}} h_{\Omega_{n, m}^{g} \rightarrow x_{m}} \\
& \prod_{n \in M_{m}^{(k)}} h_{x_{n} \rightarrow x_{m}}
\end{aligned}
$$

With Gaussian approximation, we can also calculate $p\left(\boldsymbol{x}_{m}^{(k)} \mid \tilde{\boldsymbol{O}}_{M}^{(1: k)}\right)$ using Algorithm 2.

Algorithm 2 Message calculation using unscented particle filter

Require: Initial estimate $\hat{\mu}_{x}$ and $\hat{\boldsymbol{\Sigma}}_{x}$; Distributions of all incoming messages; N particles $\left\{\boldsymbol{z}_{i}^{(0)}\right\}_{i=1}^{N} \sim p\left(\boldsymbol{x}^{(0)}\right) ; \forall i$, $\boldsymbol{z}_{i}^{(0)}=\left[\left(\tilde{\boldsymbol{z}}_{i}^{(0)}\right)^{T}, \mathbf{0}, \mathbf{0}\right]^{T}, \tilde{\boldsymbol{z}}_{i}^{(0)}=E\left[\boldsymbol{z}_{i}^{(0)}\right], \boldsymbol{\Sigma}_{i}^{(0)}=E\left[\left(\boldsymbol{z}_{i}^{(0)}-\right.\right.$ $\left.\left.\tilde{\boldsymbol{z}}_{i}^{(0)}\right)\left(\boldsymbol{z}_{i}^{(0)}-\tilde{\boldsymbol{z}}_{i}^{(0)}\right)^{T}\right], w_{i}^{(0)}=1$.

Ensure: Updated $\hat{\boldsymbol{\mu}}_{x}, \hat{\boldsymbol{\Sigma}}_{x}$ after product

1: for $i$ from 1 to $\mathrm{N}$ do

Update the particle with UKF:

- Calculate $2 n_{a}+1$ sigma points:

$\boldsymbol{Z}_{i}^{(k-1)}=\left[\tilde{\boldsymbol{z}}_{i}^{(k-1)}, \tilde{\boldsymbol{z}}_{i}^{(k-1)} \pm \sqrt{\left(n_{a}+\eta\right) \boldsymbol{\Sigma}_{i}^{(k-1)}}\right]$

- Propagate the particle into future:

$Z_{i}^{(k \mid k-1)}=h_{s}\left(Z_{i}^{(k-1)}\right)$

$\tilde{\boldsymbol{z}}_{i}^{(k \mid k-1)}=\sum_{j=0}^{2 n_{a}} W_{j}^{c 0} Z_{i}^{(j, k \mid k-1)}$

8: $\quad \boldsymbol{\Sigma}_{i}^{(k \mid k-1)}=\sum_{j=0}^{2 n_{a}} W_{j}^{c 1}\left[\boldsymbol{Z}_{i}^{(j, k \mid k-1)}-\tilde{\boldsymbol{z}}_{i}^{(k \mid k-1)}\right]$

$\left[\boldsymbol{Z}_{i}^{(j, k \mid k-1)}-\tilde{\boldsymbol{z}}_{i}^{(k \mid k-1)}\right]^{T}$

$Y_{i}^{(k \mid k-1)}=h_{m}\left(Z_{i}^{(k \mid k-1)}, \tilde{\boldsymbol{z}}_{i}^{(k-1)}\right)$

10: $\quad \tilde{y}_{i}^{(k \mid k-1)}=\sum_{j=0}^{2 n_{a}} W_{j}^{c 0} Y_{i}^{(j, k \mid k-1)}$

11: -Incorporate new observations:

12: $\quad \boldsymbol{\Sigma}_{\tilde{y}_{k}, \tilde{y}_{k}}=\sum_{j=0}^{2 n_{a}+1} W_{j}^{c 1}\left[Y_{i}^{(j, k \mid k-1)}-\tilde{y}_{i}^{(k \mid k-1)}\right]$

$\left[Y_{i}^{(j, k \mid k-1)}-\tilde{y}_{i}^{(k \mid k-1)}\right]^{T}$

13: $\quad \boldsymbol{\Sigma}_{\tilde{\boldsymbol{z}}_{k}, \tilde{y}_{k}}=\sum_{j=0}^{2 n_{a}+1} W_{j}^{c 1}\left[\boldsymbol{Z}_{i}^{(j, k \mid k-1)}-\tilde{\boldsymbol{z}}_{i}^{(k \mid k-1)}\right]$

$\left[Y_{i}^{(j, k \mid k-1)}-\tilde{y}_{i}^{(k \mid k-1)}\right]^{T}$

14: $\quad K_{k}=\Sigma_{\tilde{z}_{k}, \tilde{y}_{k}} \boldsymbol{\Sigma}_{\tilde{y}_{k}, \tilde{y}_{k}}^{-1}$

15: $\quad \boldsymbol{z}_{i}^{(k)}=\tilde{\boldsymbol{z}}_{i}^{(k \mid k-1)}+K_{k}\left(y^{(k)}-\tilde{y}_{i}^{(k \mid k-1)}\right)$

16: $\quad \hat{\boldsymbol{\Sigma}}_{i}^{(k)}=\boldsymbol{\Sigma}_{i}^{(k \mid k-1)}-K_{k} \boldsymbol{\Sigma}_{\tilde{y}_{k}, \tilde{y}_{k}}^{-1} K_{k}^{T}$

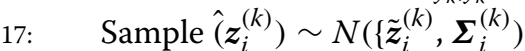

18: $\quad$ Calculate $q\left(z_{i}^{(k)}\right)$ according to Equation 18;

19: $\quad$ Evaluate the p.d.f.s at the new particle $p_{j^{\prime}}\left(z_{i}^{(k)}\right)$ for each factor using Equations 21, 22, 28, 29, 24, 30, and 25

20: Evaluate the importance weights of the new particle: $w_{i}^{(k)}=\Pi_{j^{\prime}} p_{j^{\prime}}\left(\boldsymbol{z}_{i}^{(k)}\right) / q\left(\boldsymbol{z}_{i}^{(k)}\right)$

21: end for

22: Normalize the importance weights of all particles so that $\Sigma_{i} w_{i}^{(k)}=1$

23: Estimate new mean and covariance using weighted particles:

24: $\hat{\boldsymbol{\mu}}_{x}=\sum_{i=1}^{N} w_{i}^{(k)} \boldsymbol{z}_{i}^{(k)}$

25: $\hat{\boldsymbol{\Sigma}}_{x}=\boldsymbol{\Sigma}_{i=1}^{N} w_{i}^{(k)}\left(\boldsymbol{z}_{i}^{(k)}-\hat{\boldsymbol{\mu}_{x}}\right)^{T}\left(\boldsymbol{z}_{i}^{(k)}-\hat{\boldsymbol{\mu}_{x}}\right)$

26: Resample with replacement $N$ particles $\left\{z_{i}^{(k)}\right\}_{i=1}^{N}$ from all particles $\left\{\boldsymbol{z}_{i}^{(k)}\right\}_{i=1}^{N}$ according to $\left\{w_{i}^{(k)}\right\}_{i=1}^{N}$

27: Save $\left\{\boldsymbol{z}_{i}^{(k)}\right\}_{i=1}^{N}$ and their corresponding $\left\{\boldsymbol{\Sigma}_{i}^{(k)}\right\}_{i=1}^{N}$ for next call 


\subsection{Calculating the factor products}

The products of factors, i.e., Equations 25, 26, and 41, are mathematically intractable. This is a problem that permeates in many disciplines of sciences. The most widely known methods are importance sampling [31], bootstrap particle filter [34], and unscented particle filters [35,36]. While importance sampling is convenient and attractive, it suffers from the sample degeneracy problem. Bootstrap filter and unscented particle filter try to avoid this degeneracy by context-aware resampling, which eliminates the particles having low importance weights and proliferates particles having high importance weights. A bootstrap particle filter uses state update information for resampling, while an unscented particle filter further improves the bootstrap particle filter by estimating the first- and second-order moments of the new state incorporating new observations using unscented transform before resampling.

We present the variation of unscented particle filter for calculating the factor products of this work in Algorithm 2. In Algorithm 2, $n_{a}, \eta, W_{j}^{c 0}$, and $W_{j}^{c 1}$ are parameters related to unscented transform. $n_{a}=7$ is the number of states, which is 7 in our case. $\eta=3 \alpha^{2}-n_{a}$, $W_{0}^{c 0}=\eta /\left(n_{a}+\eta\right)$, and $W_{0}^{c 1}=\eta /\left(n_{a}+\eta\right)+\left(3-\alpha^{2}\right)$ for Gaussian distributions. For $j=1$ to $2 n_{a}, W_{j}^{c 0}=W_{j}^{c 1}=$ $1 /\left[2\left(n_{a}+\eta\right)\right]$.

\section{Simulations and discussions}

\subsection{Setup}

For belief propagation combined with varied linear and nonlinear filters is widely available in the literature, we focused on evaluating the robustness of BOPU with some discussions on the appropriateness of the approximations in BOPU. Simulations are conducted by MATLAB with a fictitious scenario as given in Figure 3. In Figure 3, a group of six UAVs indexed by 0 to 5 are flying in a formation from a start point under the control of ground station $N_{0^{\prime}}$ to a destination with ground station $N_{1^{\prime}}$ and a SoOP source $G_{0}$ in view, but experience a GPS countermeasure en route.
The positions of satellites in view are given in Table 1. All UAVs follow the same velocity but with a different point of departure as also given in Table 1 . The velocity of the formation flying is given in Figure $4 \mathrm{a}$, and the route of node 0 is given in Figure $4 \mathrm{~b}$. The simulated cases include the following:

- Case 0: Whenever a node has at least four satellites in view, it will not participate in the belief propagation but will offer the statistics of its own position. In addition, the measurements with the two ground control stations $N_{0^{\prime}}$ and $N_{1^{\prime}}$ and the SoOP source $G_{0}$ signal are not utilized. Case 0 reduces the positioning traffic over the wireless network to a minimum but is expected to have the poorest positioning performance.

- Case 1: All nodes take part in the belief propagation process as stated in Algorithm 1 but do not utilize the measurements with the two ground control stations $N_{0^{\prime}}$ and $N_{1^{\prime}}$ and the SoOP source $G_{0}$ signal.

- Case 2: All nodes take part in the belief propagation process as stated in Algorithm 1, and the measurements with the two ground control stations $N_{0^{\prime}}$ and $N_{1^{\prime}}$ and the SoOP source $G_{0}$ signal are used.

- Case 3: All nodes take part in the belief propagation process. Besides, the control stations also provide pseudorange differential corrections at each epoch and broadcast to other nodes. The differential corrections help effectively remove $b_{s}^{(k)}$.

In the simulations, ground control stations 0 and 1 are placed at $\operatorname{ENU}(0,0,10)$ and $\operatorname{ENU}(50,000,50,000,10)$, respectively. The raw pseudorange observation error power $\sigma_{s \rightarrow M}^{2}=(3 \mathrm{~m})^{2}$. The mean of $b_{s}^{(k)}$ is $6 \mathrm{~m}$, and the error power of $b_{s}^{(k)}$ is $(1 \mathrm{~m})^{2}$. The error power of $\lambda(\delta f)$ is $(0.2 \mathrm{~m} / \mathrm{s})^{2}$, the error power of $\lambda(\delta \phi) /(\delta t)$ is $(0.1 \mathrm{~m} / \mathrm{s})^{2}$, the ranging error power with peers and ground control stations is $(3 \mathrm{~m})^{2}$, and the TDoA measurement error power is set to $(3 \mathrm{~m})^{2}$, with $\alpha=0.5$. The SoOP source

Table 1 Satellites and UAV departure points in the simulation setup, where $\operatorname{ENU}(0,0,0)$ corresponds to LLH(116.3328,40.0018,100)

\begin{tabular}{|c|c|c|c|c|c|c|}
\hline & 0 & 1 & 2 & 3 & 4 & 5 \\
\hline \multicolumn{7}{|c|}{ Satellite } \\
\hline$x[s]$ & $26,559,995$ & $-13,815.86$ & $-26,559,995$ & $13,815.86$ & $13,278,763$ & N/A \\
\hline$y[s]$ & $1,425.03$ & $15,232,692$ & $1,425.03$ & $15,232,692$ & $23,002,343$ & N/A \\
\hline$z[s]$ & $15,867.57$ & $21,757,722$ & $15,867.57$ & $21,757,722$ & $15,867.57$ & N/A \\
\hline \multicolumn{7}{|l|}{ User } \\
\hline$E[d]$ & 19,900 & -600 & 20,500 & 20,500 & 19,800 & 19,900 \\
\hline$N[d]$ & 19,200 & 20,100 & 20,020 & 20,500 & 19,800 & 19,900 \\
\hline$U[d]$ & 2,000 & 1,020 & 1,050 & 1,100 & 900 & 1,200 \\
\hline
\end{tabular}

N/A, not applicable. 


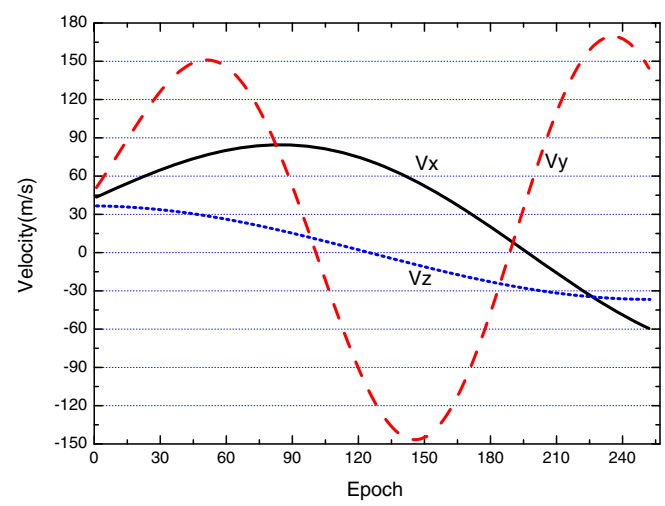

(a) Velocity of the formation flying

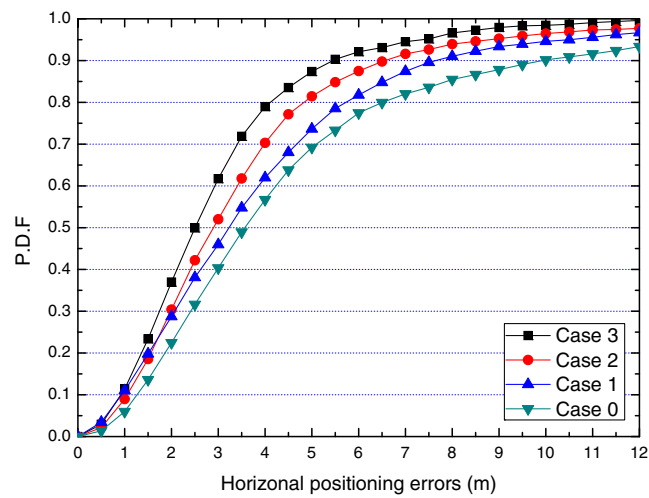

(c) Horizonal P.D.F positioning performance

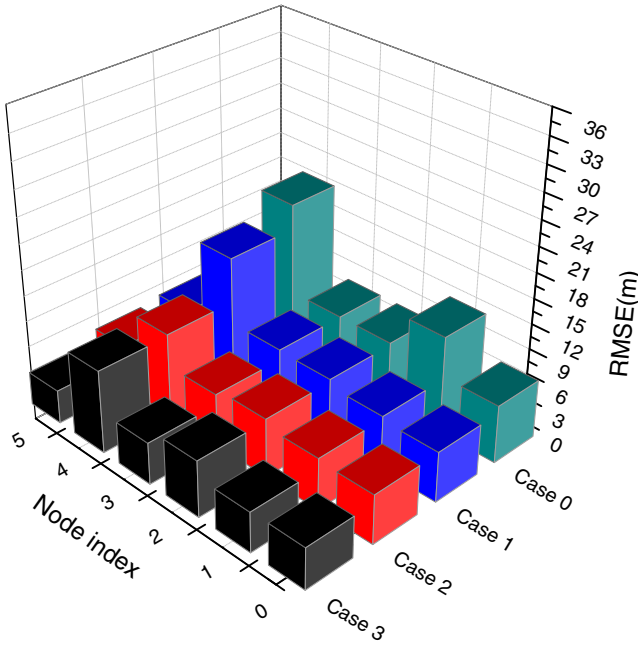

(e) Horizonal positioning RMSE

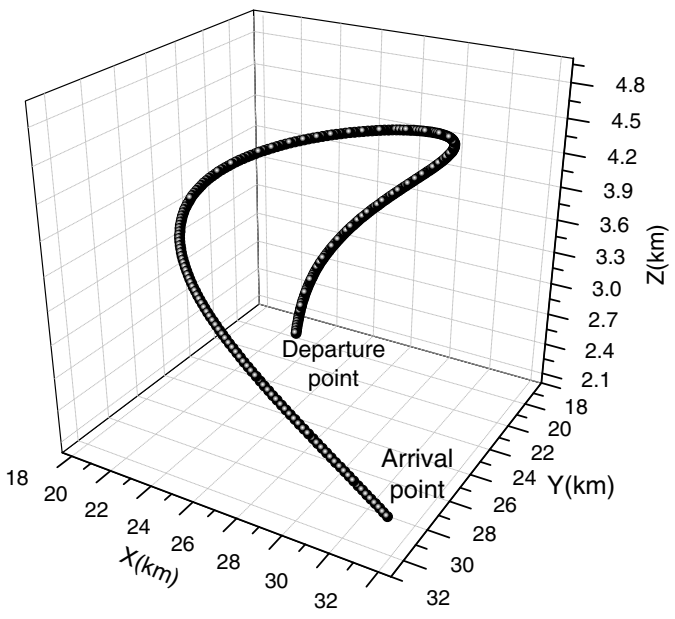

(b) The route of Node 0

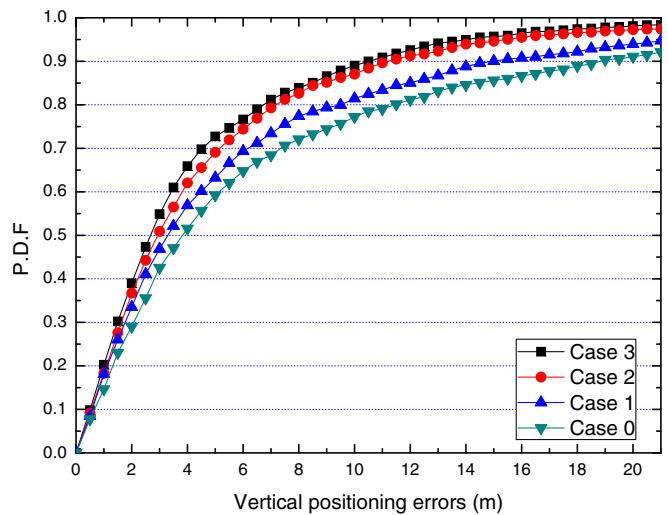

(d) Vertical P.D.F positioning performance

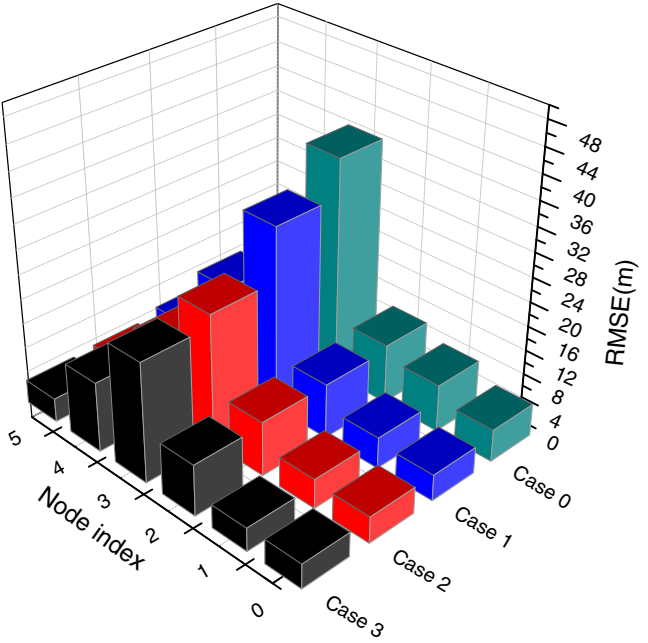

(f) Vertical positioning RMSE

Figure 4 Simulation results with ground stations $N_{0}^{\prime}$ and $N_{1}^{\prime}$. (a) Velocity of the formation flying. (b) The route of node 0. (c) Horizonal P.D.F positioning performance. (d) Vertical P.D.F positioning performance. (e) Horizonal positioning RMSE. (f) Vertical positioning RMSE. 
$G_{0}$ is placed at midway $\operatorname{ENU}(25,000,25,000,10)$. Nodes exchange time of arrival with wireless neighbors; thus, TDoA is also available between neighbors and $D_{m}^{\left(G_{0}, k\right)}=$ $R_{m}^{(k)} \backslash\left\{N_{0}^{\prime}, N_{1}^{\prime}\right\} . T_{e}=1 s$.

\subsection{Results and discussions}

Figure 4 gives the simulation results. As can be seen, from case 0 to case 3 , the positioning performance improves almost steadily. In case 0 , node 0 and node 1 have at least four satellites, and they determine their position using a traditional weighted least square positioning algorithm in order to save communication bandwidth. Without iterations with node 0 and node 1 , the positioning performance of other nodes is being restrained. In case 1 , all nodes take part in the belief propagation process, which is helpful in improving the positioning performance of all nodes (especially nodes 2 to 5), so the overall performance of case 1 is better than that of case 0 . The outperformance of case 2 over case 1 comes from the full usage of all available observations, especially ranges and closed-loop Doppler with ground control stations, and TDoA observations from SoOP source $G_{0}$. The measurements with the ground control stations $N_{0^{\prime}}, N_{1^{\prime}}$ and SoOP source $G_{0}$ help improve geometric dilution of positioning in a big way. In case 3, ground control stations also generate corrections for pseudoranges, which directly improves the quality of pseudoranges, thus the positioning precision.

The positioning performance of all nodes is given in Figure $4 \mathrm{e}, \mathrm{f}$ in terms of root mean square error (RMSE). It follows the fact that the more observables, the better precision. In case 0 , node 1 uses only the observation from four satellites in view, so its horizonal RMSE is even less than those of node 2 and node 3 . Nodes 2 to 5 , which have less than four satellites in view under some given GNSS interference, can still achieve positioning by utilizing the peer to peer measurements and the measurements with control stations. Node 5 experienced the strongest interference. Without any satellite in view but with a better geometric position, node 5 achieved even better positioning performance than nodes 3 and 4 that have satellites in view.

The results show that the performance of BOPU varies only slightly with different measurements availability. The main approximations made in the proposed BOPU are as follows: (1) The correlated errors of pseudoranges $b_{s}^{(k)}$ are ignored in factorization. The simulation results of case 3 and case 2 show that such an approximation is acceptable. (2) The dead-reckon message (19) and (20) actually ignored nonzero off-diagonal values, and Equation 11 ignores the smoothing of $\tilde{\boldsymbol{O}}_{M}^{(k-1)}$ on $\boldsymbol{x}_{m}^{(k-2)}$. Such approximations hold where the quality of observations dominates the positioning performance such as the cases in simulations. For UAVs, the positioning result using all wireless sources is further coupled with IMU measurements to reach out for a better final result.

\subsection{Algorithmic complexity}

Given a node $m$ at epoch $k$, we have one dead-reckon message, $\left|\boldsymbol{S}_{m}^{(k)}\right|$ satellite pseudorange factor messages, $\left|\boldsymbol{M}_{m}^{(k)}\right|$ messages from range neighbors, and $\sum_{g \in \boldsymbol{G}}\left|\boldsymbol{G}_{m}^{(g, k)}\right|$ messages from SoOP neighbors. It uses $N$ particles in UPF and $I$ iterations in the product estimate. The core of UPF is UKF whose complexity is $O\left(n_{a}^{3}\right)$ [37], where $n_{a}$ is the number of states as stated before. The complexities of main computations in BOPU are listed in Table 2. As was shown in Table 2, the complexity of BOPU is dominated by message multiplications needed by messages to peers, which scales as $O\left(I N\left|\boldsymbol{S}_{m}^{(k)}\right|\left(1+\left|\boldsymbol{M}_{m}^{(k)}\right|+\left|\boldsymbol{S}_{m}^{(k)}\right|+\sum_{g \in \boldsymbol{G}}\left|\boldsymbol{G}_{m}^{(g, k)}\right|\right) n_{a}^{3}\right)$.

\section{Conclusions}

Nowadays, UAVs are playing an increasingly important role both in the military and in civil affairs. Worries on the robustness of GNSS have also been increasing with the maturity of GNSS countermeasures and proliferation of wireless devices. With the aim of providing a better navigator for UAVs, we investigated the positioning of UAVs with all wireless sources via belief propagation with unscented particle filtering. By jointly using the measurements from GNSS satellites, peer UAVs, ground control stations, and signal of opportunities, the proposed algorithm, which is named BOPU, provides an improved positioning robustness and algorithmically proven high precision. By being opportunistic, BOPU allows for not only flexible variations of measurements availability but also agile compromise between wireless bandwidth consumption and positioning performance when put into practice.

\section{Table 2 Computational complexity of node $m$ in the} proposed BOPU

\begin{tabular}{|c|c|c|}
\hline Computation & Complexity & $\begin{array}{c}\text { Iteration/ } \\
\text { epoch }\end{array}$ \\
\hline $\begin{array}{l}\text { Dead-reckon } \\
\text { message }\end{array}$ & $O\left(n_{a}+n_{a}^{2}\right)$ & 1 \\
\hline $\begin{array}{l}\text { Satellite factor } \\
\text { message }\end{array}$ & $\mathrm{O}\left|\boldsymbol{S}_{m}^{(k)}\right| n_{a}$ & 1 \\
\hline $\begin{array}{l}\text { Carrier phase factor } \\
\text { message }\end{array}$ & $\mathrm{O}\left|\boldsymbol{S}_{m}^{(k)}\right| n_{a}$ & 1 \\
\hline Message to peers & $\begin{array}{l}O\left(N\left|\boldsymbol{S}_{m}^{(k)}\right|\left(1+\left|\boldsymbol{M}_{m}^{(k)}\right|+\left|\boldsymbol{S}_{m}^{(k)}\right|\right.\right. \\
\left.\left.+\sum_{g \in \boldsymbol{G}}\left|\boldsymbol{G}_{m}^{(g, k)}\right|\right) n_{a}^{3}\right)\end{array}$ & 1 \\
\hline $\begin{array}{l}\text { Message from range } \\
\text { neighbors }\end{array}$ & $\left.O\left(\left|\boldsymbol{M}_{m}^{(k)}\right|\right) n_{a}^{3}\right)$ & 1 \\
\hline $\begin{array}{l}\text { Message from SoOP } \\
\text { neighbors }\end{array}$ & $\left.O\left(\sum_{g \in \boldsymbol{G}}\left|\boldsymbol{G}_{m}^{(g, k)}\right|\right) n_{a}^{3}\right)$ & 1 \\
\hline Compute $p\left(x_{m}^{(k)} \mid \tilde{\boldsymbol{O}}_{M}^{(1: k)}\right)$ & $\begin{array}{l}O\left(N\left(1+\left|\boldsymbol{M}_{m}^{(k)}\right|+\left|\boldsymbol{S}_{m}^{(k)}\right|\right.\right. \\
\left.\left.+\sum_{g \in \boldsymbol{G}}\left|\boldsymbol{G}_{m}^{(g, k)}\right|\right) n_{a}^{3}\right)\end{array}$ & 1 \\
\hline
\end{tabular}




\section{Competing interests}

The authors declare that they have no competing interests.

\section{Received: 27 May 2013 Accepted: 17 September 2013} Published: 25 September 2013

\section{References}

1. J Blanch, TWalter, P Enge, Satellite navigation for aviation in 2025 Proc. IEEE. 100, 1821-1830 (2012)

2. R Garello, L Lo Presti, P di Torino, J Samson, Peer-to-peer cooperative positioning part I: GNSS-aided acquisition. Inside GNSS 55-63 (March/April 2012)

3. S Pullen, X Gao, GNSS jamming in the name of privacy-potential threat to GPS aviation. Inside GNSS 34-41 (March/April 2012)

4. A Dammann, S Sand, R Raulefs, in Proceedings of the 20th European Signal Processing Conference (EUSIPCO). Signals of opportunity in mobile radio positioning (Bucharest, 27-31 Aug 2012), pp. 549-553

5. LP Gill, D Grenier, JY Chouinard, Use of XM radio satellite signal as a source of opportunity for passive coherent location. IET Radar, Sonar Navigation. 5(5), 536-544 (2011)

6. M Robinson, R Ghrist, Topological localization via signals of opportunity. IEEE Trans. Signal Process. 60(5), 2362-2373 (2012)

7. J Bao-Yen Tsui, Fundamentals of Global Positioning System Receivers: A Software Approach (Wiley, New York, 2004)

8. SJ Julier, JK Uhlmann, HF Durrant-Whyten, in Proceedings of the American Control Conference, A new approach for filtering nonlinear systems. Seattle, 21-23 June 1995, pp. 1628-1632

9. TPerala, R Piche, in Proceedings of the 4th Workshop on Positioning Navigation and Communication (WPNC). Robust extended Kalman filtering in hybrid positioning applications (Hannover, 22-22 March 2007), pp. 55-63

10. EA Wan, R Van Der Merwe, in Proceedings of the IEEE Adaptive Systems for Signal Processing, Communications, and Control Symposium (AS-SPCC). The unscented Kalman filter for nonlinear estimation (Alberta, 1-4 October 2000), pp. 153-158

11. X Zhai, F Qi, H Zhang, in Proceedings of the Symposium on Photonics and Optoelectronics (SOPO). Application of unscented Kalman filter in GPS/INS (Shanghai, 21-23 May 2012), pp. 1-3

12. S Sarkka, On unscented Kalman filtering for state estimation of continuous-time nonlinear systems. IEEE Trans. Automatic Control. 52(9), 1631-1641 (2007)

13. H Wymeersch, J Lien, MZ Win, Cooperative localization in wireless networks. Proc. IEEE. 972, 427-450 (2009)

14. N Alam, A Tabatabaei Balaei, AG Dempster, A DSRC Doppler-based cooperative positioning enhancement for vehicular networks with GPS availability. IEEE Trans. Vehicular Technol. 60(9), 4462-4470 (2011)

15. MA Caceres, F Sottile, R Garello, MA Spirito, in Proceedings of the Personal, Indoor and Mobile Radio Communications (PIMRC). Hybrid GNSS-ToA localization and tracking via cooperative unscented Kalman filter (Istanbul, 26-30 Sept 2010), pp. 272-276

16. J Yao, AT Balaei, M Hassan, N Alam, AG Dempster, Improving cooperative positioning for vehicular networks. IEEE Trans. Vehicular Technol. 60(6), 2810-2823 (2011)

17. M Gholami, S Gezici, E Strom, Improved position estimation using Hybrid TW-TOA and TDOA in cooperative networks. IEEE Trans. Signal Process. 60(7), 3770-3785 (2012)

18. Y Qu, Q Tian, in Proceedings of the International Conference on Computational Aspects of Social Networks (CASON). Multi-UAV cooperative positioning based on Delaunay triangulation (Taiyuan, 26-28 Sept 2010), pp. 401-404

19. Y Qu, Y Zhang, in Proceedings of the IEEE/ASME International Conference on Mechatronics and Embedded Systems and Applications (MESA). Fault-tolerant localization for multi-UAV cooperative flight (Qingdao, 15-17 July 2010), pp. 131-136

20. Y Qu, Y Zhang, Cooperative localization against GPS signal loss in multiple UAVs flight. J. Syst. Eng. Electron. 22(1), 103-112 (2011)

21. Z Xiong, F Sottile, MA Caceres, R Garello, in Proceedings of the IEEE-APS Topical Conference on Antennas and Propagation in Wireless Communications (APWC). Hybrid WSN-RFID cooperative positioning based on extended Kalman filter (Torino, 12-16 Sept 2011), pp. 990-993
22. N Wang, LQ Yang, in Proceedings of the 24th International Technical Meeting of the Satellite Division of the Institute of Navigation (ION GNSS). Semidefinite programming for GPS (Oregon, 21-23 Sept 2011), pp. 2120-2126

23. A So, YY Ye, Theory of semidefinite programming for sensor network localization. Math. Program. 109, 367-384 (2007)

24. S Severi, G Abreu, G Destino, D Dardari, in Proceedings of the IEEE Global Telecommunications Conference (GLOBECOM), Understanding and solving flip-ambiguity in network localization via semidefinite programming. Honolulu, 30 Nov-4 Dec 2009, pp. 1-6

25. WY Chiu, BS Chen, CY Yang, Robust relative location estimation in wireless sensor networks with inexact position problems. IEEE Trans. Mobile Comput. 11, 935-946 (2012)

26. AY Alfakih, MF Anjos, V Piccialli, H Wolkowicz, Euclidean distance matrices, semidefinite programming and sensor network localization. Portugaliae Mathematica, 53-102 (2011)

27. H Waki, S Kim, M Kojima, M Muramatsu, Sums of squares and semidefinite program relaxations for polynomial optimization problems with structured sparsity. Siam J. Optimization. 17(1), 218-242 (2006)

28. MA Caceres, F Penna, H Wymeersch, R Garello, Hybrid cooperative positioning based on distributed belief propagation. IEEE J. Selected Areas Commun. 29(10), 1948-1958 (2011)

29. F Sottile, H Wymeersch, MA Caceres, MA Spirito, in Proceedings of the IEEE Global Telecommunications Conference (GLOBECOM). Hybrid GNSS-terrestrial cooperative positioning based on particle filter (Houston, 5-9 Dec 2011), pp. 5-9

30. FR Kschischang, BJ Frey, HA Loeliger, Factor graphs the sum-product algorithm. IEEE Trans. Inf. Theory. 47(2), 498-519 (2001)

31. D Barber, Bayesian Reasoning and Machine Learning (Cambridge University Press, Cambridge, 2012)

32. WM Smith, DC Cox, in Proceedings of the IEEE Antennas and Propagation Society International Symposium (APSURSI). A closed-loop Doppler measurement for velocity estimation in mobile, multipath environments (Monterey, 20-25 June 2004), pp. 2203-2206

33. RR Hatch, in Proceedings of the 3rd International Symposium on Satellite Doppler Positioning. The synergism of GPS code and carrier measurements (New Mexico, 8-12 Feb 1982), pp. 1213-1231

34. NJ Gordon, DJ Salmond, AFM Smith, Novel approach to nonlinear/non-Gaussian Bayesian state estimation. IEEE Proc. Radar Signal Process. 140(2), 107-113 (1993)

35. W Guo, C Han, M Lei. Improved unscented particle filter for nonlinear Bayesian estimation (Quebec, 9-12 July 2007), pp. 1-12

36. HW Li, J Wang, HT Su, Improved particle filter based on differential evolution. Electron. Lett. 47(19), 1078-1079 (2011)

37. S Haykin, Kalman Filtering And Neural Networks (Wiley, New York, 2011), pp. 243-279

\section{doi:10.1186/1687-6180-2013-150}

Cite this article as: Chen et al:: Robust all-source positioning of UAVs based on belief propagation. EURASIP Journal on Advances in Signal Processing 2013 2013:150.

\section{Submit your manuscript to a SpringerOpen ${ }^{\circ}$ journal and benefit from:}

- Convenient online submission

- Rigorous peer review

- Immediate publication on acceptance

- Open access: articles freely available online

- High visibility within the field

Retaining the copyright to your article

Submit your next manuscript at $>$ springeropen.com 